\title{
Cutaneous T-cell Lymphoma and Concomitant Atopic Dermatitis Responding to Dupilumab
}

\author{
Nicholas K. Mollanazar, MD, MBA; Kevin T. Savage, MD; Bobak T. Pousti, MD; Neha Jariwala, MD; \\ Christina Del Guzzo, MD; Paul Haun, MD, MS; Carmela C.Vittorio, MD; Alain H. Rook, MD; Ellen J. Kim, MD
}

\section{PRACTICE POINTS}

- The diagnosis of cutaneous T-cell lymphoma (CTCL), particularly early-stage disease, remains challenging and often requires a combination of serial clinical evaluations as well as laboratory diagnostic examinations.

- Dupilumab and its effect on helper $T_{\text {cell }}\left(T_{H} 2\right)$ skewing may play a role in the future management of CTCL.
Cutaneous T-cell lymphoma (CTCL) represents a diagnostic challenge because of its large symptomatic overlap with other common skin conditions such as atopic dermatitis (AD) and psoriasis. Dupilumab has offered promising results in AD treatment; however, concerns exist that its use may exacerbate undiagnosed CTCL. We present a patient with $C T C L$ and concomitant $A D$ who experienced improvement in both CTCL blood involvement and AD following the addition of dupilumab therapy.

Cutis. 2020;106:131-132.

$\square^{2}$ atients with cutaneous T-cell lymphoma (CTCL) often are diagnosed with atopic dermatitis (AD) or psoriasis before receiving their CTCL diagnosis. The effects of new biologic therapies for $\mathrm{AD}$ such as dupilumab, an IL-4/IL-13 antagonist, on CTCL are unknown. Dupilumab may be beneficial in CTCL given that helper $\mathrm{T}$ cell $\left(\mathrm{T}_{\mathrm{H}} 2\right)$ cytokines are increased in advanced CTCL. ${ }^{1}$ We present a patient with definitive CTCL and concomitant $\mathrm{AD}$ who was safely treated with dupilumab and experienced improvement in both CTCL and AD.

\section{Case Report}

A 68-year-old man presented with increased itching from $\mathrm{AD}$ and a new rash on the arms, neck, chest, back, and lower extremities (Figures 1A and 2A). He had a medical history of $\mathrm{AD}$ and $\mathrm{CTCL}$ diagnosed by biopsy and peripheral blood flow cytometry (stage IVA1 [T4N0M0B2]) that was being treated with comprehensive multimodality therapy consisting of bexarotene $375 \mathrm{mg}$ daily, interferon alfa- $2 \mathrm{~b}$ 3 mIU 3 times weekly, interferon gamma-1b 2 mIU 3 times weekly, total skin electron beam therapy followed by narrowband UVB twice weekly, and extracorporeal photopheresis every 4 weeks, which resulted in a partial clinical response for 6 months. A biopsy performed at the current presentation showed focal spongiosis and features of lichen simplex chronicus with no evidence suggestive of CTCL. Peripheral blood flow cytometry showed stable $\mathrm{B}_{1}$-staged disease burden (CD4/CD8, 2.6:1); $\mathrm{CD}^{+} / \mathrm{CD}^{-}, 12 \%$ [91/ $\left.\mu \mathrm{L}\right]$; $\left.\mathrm{CD}^{+} / \mathrm{CD} 26^{-}, 21 \%[155 / \mu \mathrm{L}]\right)$. Treatment with potent and superpotent topical steroids was attempted for more than 6 months and was unsuccessful in relieving the symptoms.

Given the recalcitrant nature of the patient's rash and itching, dupilumab was added to his CTCL regimen. Prior to initiating dupilumab, the patient reported a numeric rating scale itch intensity of 7 out of 10 . After 4 weeks of treatment with dupilumab, the patient reported a numeric rating scale itch intensity of 1 . Over a 3 -month period, the patient's rash improved dramatically (Figures 1B and 2B), making it possible to decrease CTCL treatmentsbexarotene decreased to $300 \mathrm{mg}$, interferon alfa- $2 \mathrm{~b}$ to $3 \mathrm{mIU}$ twice weekly, interferon gamma- $1 \mathrm{~b}$ to $2 \mathrm{mIU}$ twice weekly, extracorporeal photopheresis every 5 weeks, and

Drs. Mollanazar and Pousti are from the Department of Dermatology, Lewis Katz School of Medicine at Temple University, Philadelphia, Pennsylvania. Dr. Savage is from Drexel University College of Medicine, Philadelphia. Drs. Jariwala, Del Guzzo, Haun, Vittorio, Rook, and Kim are from the Department of Dermatology, Perelman School of Medicine, University of Pennsylvania, Philadelphia.

Dr. Mollanazar is an investigator for Sanofi and Regeneron Pharmaceuticals Inc. Drs. Savage, Pousti, Jariwala, Del Guzzo, Haun, Vittorio, Rook, and Kim report no conflict of interest.

Correspondence: Bobak T. Pousti, MD, Department of Dermatology, Lewis Katz School of Medicine at Temple University, 3322 N Broad St, Medical Office Building, Ste 212, Philadelphia, PA 19140 (bobak.pousti@temple.edu).

doi: 10.12788 /cutis.0066 
FIGURE 1. A, Upper arm of a patient with cutaneous T-cell lymphoma and atopic dermatitis before treatment. B, Following 3 months of dupilumab therapy.
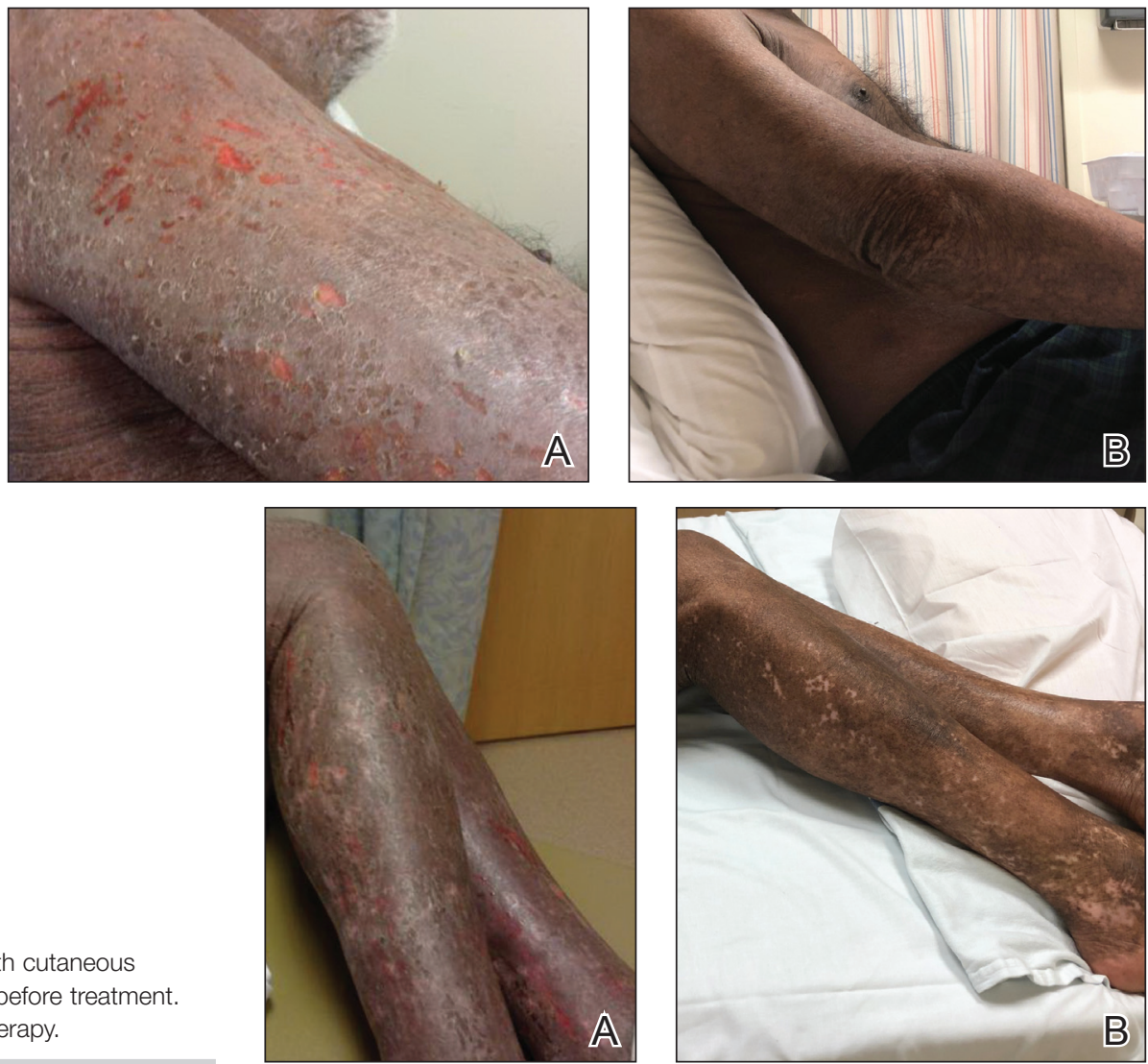

FIGURE 2. A, Lower leg of a patient with cutaneous T-cell lymphoma and atopic dermatitis before treatment. $\mathrm{B}$, Following 3 months of dupilumab therapy. narrowband UVB was discontinued completely. A comparison of the patient's flow cytometry analysis from before treatment to 3 months after dupilumab showed an overall slight reduction in CTCL $B_{1}$ blood involvement and normalization of the patient's absolute eosinophil count and serum lactate dehydrogenase level. The patient tolerated the treatment well without any adverse events and has maintained clinical response for 6 months.

\section{Comment}

Cutaneous T-cell lymphomas represent a heterogeneous group of T-cell lymphoproliferative disorders involving the skin. ${ }^{2}$ The definitive diagnosis of CTCL is challenging, as the clinical and pathologic features often are nonspecific in early disease. Frequently, undiagnosed patients are treated empirically with immunosuppressive agents. Tumor necrosis factor inhibitors and cyclosporine are both associated with progression or worsening of undiagnosed CTCL.3,4 Dupilumab was the first US Food and Drug Administrationapproved biologic for the treatment of moderate to severe AD. Cutaneous T-cell lymphoma has immunologic features, such as $\mathrm{T}_{\mathrm{H}} 2$ skewing, that overlap with $\mathrm{AD}$; however, the effects of dupilumab in CTCL are not yet known., ${ }^{5,6}$ Our group has seen patients initially thought to have AD who received dupilumab without improvement and were subsequently diagnosed with CTCL, suggesting dupilumab did not affect CTCL tumor cells. Given these findings, there was concern that dupilumab might exacerbate undiagnosed
CTCL. Our patient with definitive, severe, refractory CTCL noted marked improvement in both $\mathrm{AD}$ and underlying CTCL with the addition of dupilumab. No other treatments were added. The response was so dramatic that we were able to wean the doses and frequencies of several CTCL treatments. Our findings suggest that dupilumab may be beneficial in a certain subset of CTCL patients with a history of $\mathrm{AD}$ or known concomitant AD. Prospective studies are needed to fully investigate dupilumab safety and efficacy in CTCL and whether it has any primary effects on tumor burden in addition to benefit for itch and skin symptom relief.

\section{REFERENCES}

1. Guenova $\mathrm{E}$, Watanabe $\mathrm{R}$, Teague JE, et al. $\mathrm{T}_{\mathrm{H}} 2$ cytokines from malignant cells suppress $\mathrm{T}_{\mathrm{H}} 1$ responses and enforce a global $\mathrm{T}_{\mathrm{H}} 2$ bias in leukemic cutaneous T-cell lymphoma. Clin Cancer Res. 2013;19:3755-3763.

2. Wilcox RA. Cutaneous T-cell lymphoma: 2016 update on diagnosis, risk-stratification, and management. Am J Hematol. 2016;91:151-165

3. Martinez-Escala ME, Posligua AL, Wickless H, et al. Progression of undiagnosed cutaneous lymphoma after anti-tumor necrosis factoralpha therapy. J Am Acad Dermatol. 2018;78:1068-1076.

4. Pielop JA, Jones D, Duvic M. Transient CD30+ nodal transformation of cutaneous T-cell lymphoma associated with cyclosporine treatment. Int J Dermatol. 2001;40:505-511.

5. Saulite I,HoetzeneckerW, Weidinger S, et al. Sézary syndrome and atopic dermatitis: comparison of immunological aspects and targets [published online May 17, 2016]. BioMed Res Int. doi:10.1155/2016/9717530.

6. Sigurdsson V, Toonstra J, Bihari IC, et al. Interleukin 4 and interferongamma expression of the dermal infiltrate in patients with erythroderma and mycosis fungoides. an immuno-histochemical study. J Cutan Pathol. 2000;27:429-435. 